International Journal of Modern Physics: Conference Series

(C) World Scientific Publishing Company

\title{
Higgs Searches Beyond the Standard Model
}

\author{
R. Mankel, on behalf of the ATLAS, CMS, CDF and D0 collaborations \\ Deutsches Elektronen-Synchrotron (DESY), \\ Notkestr 85, D-22603 Hamburg, Germany \\ Rainer.Mankel@desy.de \\ Received Day Month Year \\ Revised Day Month Year
}

\begin{abstract}
While the existence of a Higgs boson with a mass near $125 \mathrm{GeV}$ has been clearly established, the detailed structure of the entire Higgs sector is yet unclear. Besides the Standard Model interpretation, various possibilities for extended Higgs sectors are being considered. The minimal supersymmetric extension (MSSM) features two Higgs doublets resulting in five physical Higgs bosons, which are subject to direct searches. Alternatively, more generic Two-Higgs Doublet models (2HDM) are used for the interpretation of results. The Next-to-Minimal Supersymmetric Model (NMSSM) has a more complex Higgs sector with seven physical states. Also exotic Higgs bosons decaying to invisible final states are considered. This article summarizes recent findings based on results from collider experiments.
\end{abstract}

Keywords: Higgs,Boson,BSM,MSSM,NMSSM,Supersymmetry,2HDM

PACS numbers:

\section{Introduction}

The electroweak symmetry breaking mechanism of the Standard Model (SM) predicts the Higgs particle as a scalar boson. The discovery of a Higgs boson with a mass near $125 \mathrm{GeV}^{112}$ by the experiments ATLAS $S^{\sqrt{3}}$ and $\mathrm{CMS}^{4}$ at the CERN Large Hadron Collider (LHC) is an important milestone, and it is of fundamental interest to study the properties of this state, its quantum numbers and couplings. An equally important aim is the unravelling of the overall structure of the Higgs sector. While at the level of current measurements, the observed state is compatible with the Higgs boson as predicted by the SM, the mass of the Higgs boson is divergent at high energies ${ }^{5}$. For many other open questions, related e.g. to dark matter, naturalness and $\mathrm{CP}$ violation in the universe, solutions might evolve through more detailed studies of the Higgs sector. From investigation of the observed boson alone one obtains relatively weak contraints on Higgs decays beyond the Standard Model (BSM); for example, a study of the CMS experiment (Figure 1 ${ }^{6}$ derives an upper limit of $52 \%$ for the branching fraction into BSM decay modes at $95 \%$ confidence level. Significant improvement of such limits can at least be expected to take a long 
time. For this reason, direct searches of BSM Higgs signatures are essential to clarify the structure of the Higgs sector.

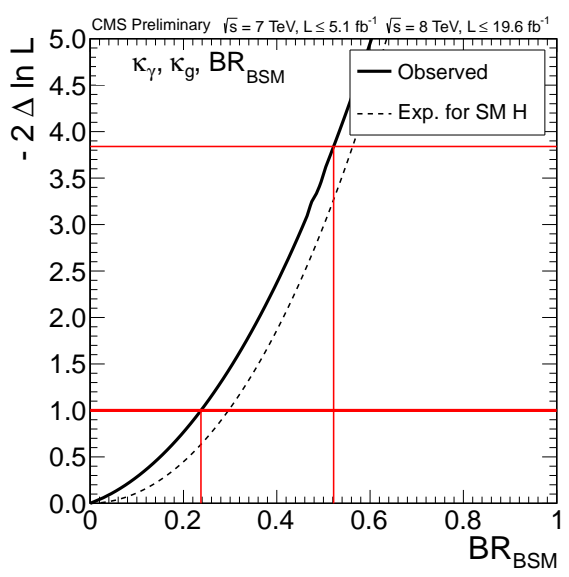

Fig. 1. 1D test statistics as a function of the Higgs branching fraction into non-SM decay modes 6 . Only the photon and gluon couplings within the loops are modified. The tree-level couplings are fixed at SM level.

\section{The MSSM Higgs Sector}

While the SM features a single complex Higgs doublet resulting in one physical scalar Higgs boson, the Minimal Supersymmetric Model (MSSM) is characterized by an extended Higgs sector with two complex Higgs doublets. This gives rise to five physical Higgs bosons, three of them neutral, labeled h, H (CP-even) and A (CP-odd) and jointly referred to as $\phi$, and the other two charged, named $\mathrm{H}^{ \pm}$. At tree level, the MSSM Higgs sector is governed by two parameters: the mass $m_{A}$ and $\tan \beta$, which is the ratio of the vacuum expectation values of the two Higgs doublets. Beyond tree level, additional parameters enter via radiative corrections, and benchmark scenarios fixing these parameters are used to compare different measurements. In many cases, the $m_{h}^{\max }$ benchmark scenarid ${ }^{7}$ is used.

The mass of the CP-odd Higgs boson is usually approximately degenerate with one of the CP-even bosons, either the $\mathrm{H}$ for large $m_{A}$, or the $\mathrm{h}$ if $m_{A}$ is small. With the exception of the di-muon channel, this degeneracy cannot be resolved within the experimental mass resolution, and thus the visible cross section effectively doubles. The coupling to the $\mathrm{b}$ quark is proportional to $\tan \beta$. Thus production cross sections in association with $\mathrm{b}$ quarks are enhanced by a factor of $\approx 2 \tan ^{2} \beta$. It is important to note that the observation of a Higgs boson near $125 \mathrm{GeV}$ with SM-like properties does not exclude additional heavy Higgs bosons at large $\tan \beta$, as discussed in detail in Ref. 8. If $m_{A}$ is large compared to the $\mathrm{Z}$ mass, the light MSSM Higgs boson (h) becomes SM-like, a situation referred to as the decoupling limit. At the current 
level of measurements, both SM and MSSM are found to fit the measurements about equally well, see for example Ref. 9 .

\subsection{MSSM searches in the $\tau \tau$ channel}

The $\phi \rightarrow \tau \tau$ channel can be seen as a good compromise between a relatively large branching fraction and manageable backgrounds. Searches in this channel have been performed by the Tevatron ${ }^{10}$ and LHC experiments; the latter will be described in more detail. The main production mechanisms are associated production with $\mathrm{b}$ quarks and gluon-gluon fusion (GGF). In correspondence, the data are sub-divided in event categories with at least one, or no b-tagged jet. The decay modes of the $\tau$ are grouped into leptonic ( $e$ or $\mu$ ) and hadronic decays ("had"). This results in six decay patterns, five of which are covered by the analyses of ATLAS ${ }^{11}$ and CMS 12 : $e+\mu, e+h a d, \mu+h a d, h a d+h a d$ (only ATLAS), $\mu+\mu$ (only CMS). The invariant mass of the $\tau$ pair is reconstructed from the visible decay products and the missing transverse energy, using a likelihood technique (CMS) and a method named Missing Mass Calculator $\frac{[13}{}$ (ATLAS).
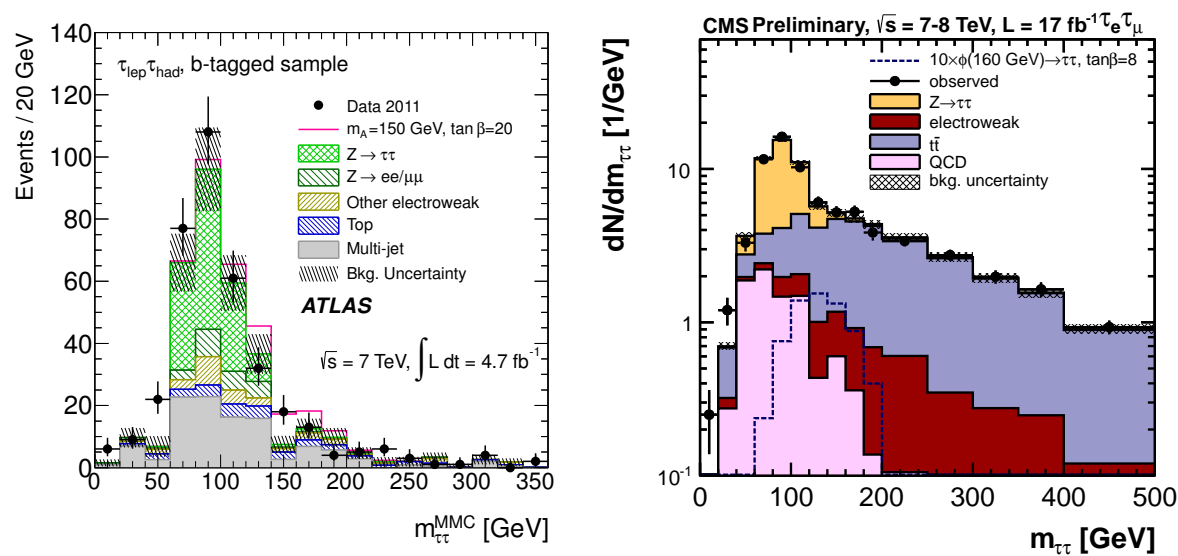

Fig. 2. Distributions of reconstructed invariant mass of the $\tau \tau$ system in the btag category; from ATLAS 11 in the combined $e+h a d$ and $\mu+$ had channels (left) and CMS12 (right) in the $e+\mu$ channel. Estimated backgrounds are also shown.

A very important background arises from $Z \rightarrow \tau \tau$ decays. It is addressed with an embedding technique: events with reconstructed $Z \rightarrow \mu \mu$ decays are taken from the data, and the muon objects are replaced with simulated $\tau$ decays. Additional backgrounds arise from $Z \rightarrow e e(\mu \mu)$ decays, $t \bar{t}$ and di-boson production, QCD multijet and $\mathrm{W}+$ jets events. Their relevance differs across the various $\tau \tau$ decay patterns. The results shown here are based on the $7 \mathrm{TeV}$ data sample in the case of ATLAS, while the CMS results include the first part of the $8 \mathrm{TeV}$ data. Figure 2 
shows examples of the reconstructed $m_{\tau \tau}$ spectra for selected decay patterns in the b-tag category for ATLAS and CMS together with the estimated backgrounds. The summed background estimates describe the data very well, and there is no indication of a signal.
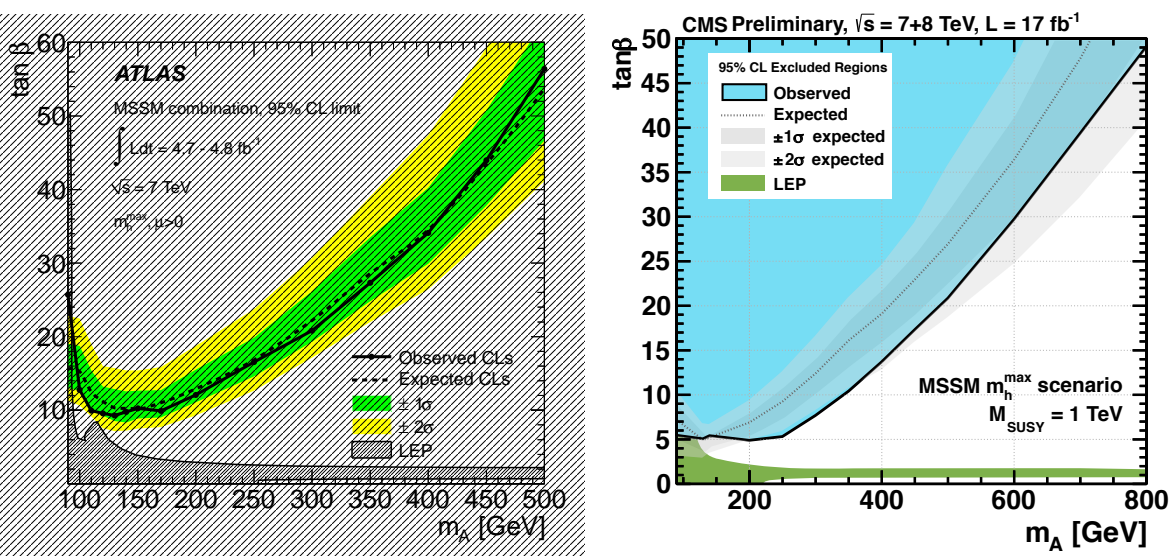

Fig. 3. Upper limits for $\tan \beta$ vs. $m_{A}$ obtained in the $\tau \tau$ channel from the ATLAS 11 (left) and CMS experiments ${ }^{12}$ (right). The ATLAS measurement also includes the $\phi \rightarrow \mu \mu$ channel.

The results from the various event categories and decay patterns are combined, and are used to compute upper limits on the MSSM parameter $\tan \beta$ at the $95 \%$ confidence level as a function of $m_{A}$. The ATLAS analysis also includes the $\phi \rightarrow$ $\mu^{+} \mu^{-}$decay channel, which is not discussed here in detail. The results are shown in Figure 3 in the $m_{h}^{\max }$ scenario. At low masses (below $m_{A} \approx 250 \mathrm{GeV}$ ), the upper limit on $\tan \beta$ reaches down to values of 5 , and touches the lower limit obtained by the LEP experiments. At larger $m_{A}$, there is still a wide range of $\tan \beta$ allowed. Inclusion of the full set of $8 \mathrm{TeV}$ data can be expected to give further improvements in the sensitivity of both experiments.

\subsection{MSSM searches in the $b \bar{b}$ channel}

In the MSSM, the Higgs decay into two b quarks is the dominating channel for $\tan \beta$ significantly larger than one. Furthermore, the associated Higgs production with $\mathrm{b}$ quarks is enhanced by a factor of $\tan ^{2} \beta$ due to the modified coupling. On the other hand, there is a copious background from QCD multi-jet production, which makes this analysis very difficult and in particular challenging from the trigger aspect.

The analyses shown here search for $\phi \rightarrow b \bar{b}$ signatures with at least one additional b-tagged jet. This channel was first successfully analyzed by the Tevatron experiments CDF and $\mathrm{D} 014 \sqrt[15 \mid 16]{ }$. The signal is searched in the invariant mass of the two leading jets, which must be b-tagged. CDF uses a second variable which 

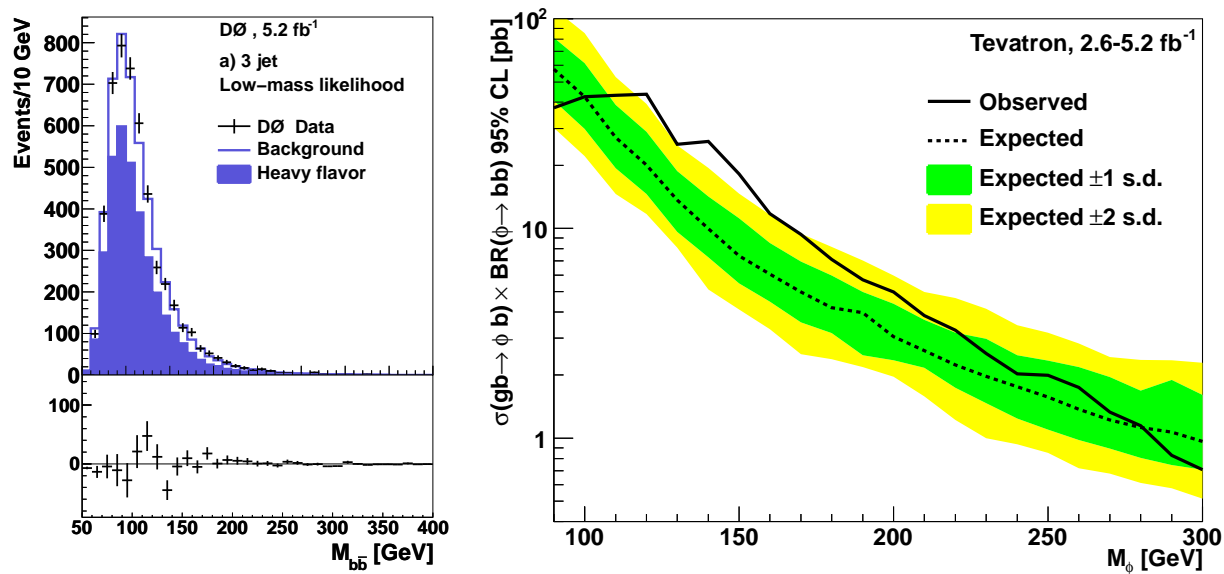

Fig. 4. Left: Invariant mass spectrum of the two leading $\mathrm{b}$ jets in triple-b-tag events from the D0 experiment, together with the estimated background 15 . A likelihood-based selection optimized for the low-mass region is shown. Right: Combined cross section times branching fraction upper limit from the $\mathrm{CDF}$ and $\mathrm{D} 0$ experiments 16 .

functions as a global b-tag. The background estimation, which is a key component of the analyses, is performed with different methods. The CDF analysis derives background templates from the double b-tag sample by applying b-tag efficiency weights. The combination of the various background templates, together with a signal template, is fitted to the data. In the D0 analysis, fractional contributions of the various multi-jet processes are determined by fitting $p_{T}$ distributions from simulation to the data. The invariant mass spectrum from the D0 analysis is shown in Figure 4 (left) 15 . The data are shown along with the data-driven background estimate. The combined upper limits on the cross section times branching fraction is shown in Figure 4 (right) 16 . Neither experiment sees a signal above the background expectation, but there are modest excesses in the observed vs. the expected upper limits of $\approx 2.8 \sigma$ in the $\mathrm{CDF}$ and of $\approx 2.5 \sigma$ in the D0 case, which are both visible in the combination plot at relatively low masses.

The first analysis of this channel at the LHC has been carried out by CMS17. The search is performed both in the all-hadronic final state with three b-tagged jets, as well as in semi-leptonic signatures requiring in addition a non-isolated muon in one of the b-tagged jets. The all-hadronic analysis is inspired by the CDF method, using a background model of templates determined from the double-b-tag sample. The invariant mass spectrum of the two leading jets is shown in Figure 5 (left). The observed spectrum can be fitted well with a combination of five background templates, and there is no indication of a signal or excess. Upper limits for the cross section times branching ratio are converted to limits in the MSSM parameter space in the $m_{h}^{\max }$ scenario. In Figure 5 (right) these limits are shown as a function of $\tan \beta$ in comparison to the combined Tevatron results. The CMS numbers have 

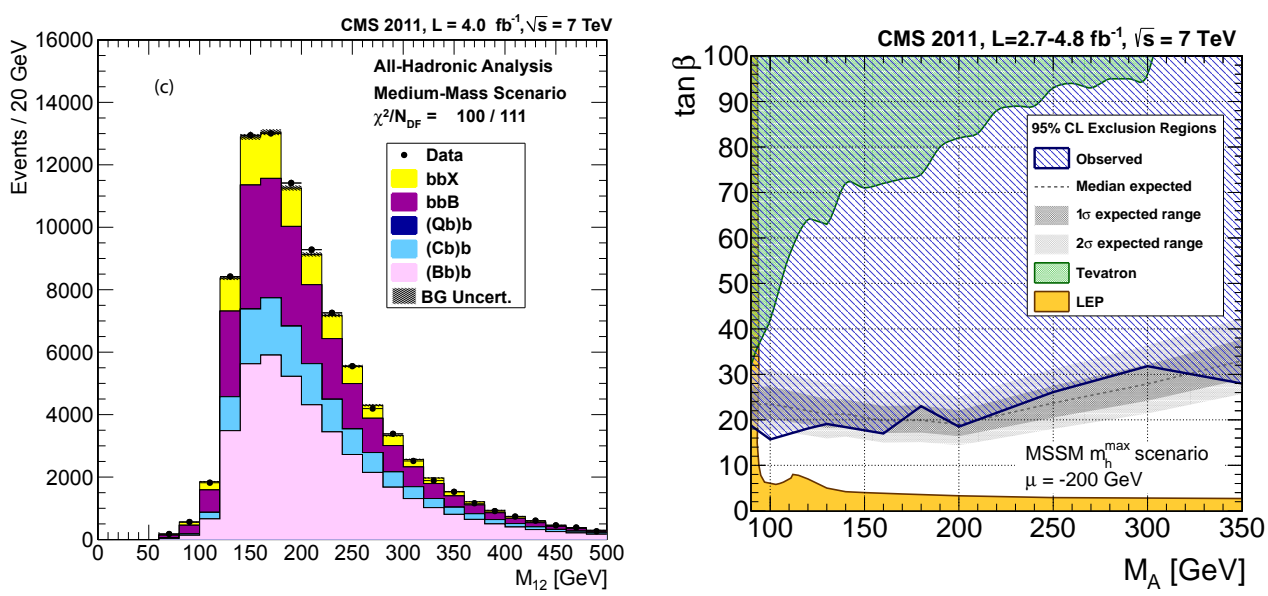

Fig. 5. Left: Invariant mass spectrum of the two leading $\mathrm{b}$ jets in triple-b-tag events from the all-hadronic analysis in CMS17. The shaded stacked histograms show the contribution of the different background templates resulting from the background-only fit. Right: Observed upper limits at $95 \%$ confidence level on $\tan \beta$ as a function of $m_{A}$ for the combined all-hadronic and semi-leptonic analyses from CMS17. Exclusion regions from LEP and Tevatron are also shown.

been converted for a Higgsino mass parameter of $\mu=-200 \mathrm{GeV}$ to allow direct comparison with the Tevatron numbers. The CMS results shows a much higher sensitivity already with the $7 \mathrm{TeV}$ data, with limits ranging between 18 and 32 in the whole mass range up to $350 \mathrm{GeV}$.

\subsection{Charged Higgs boson search}

Discovery of a charged Higgs boson would be an immediate indication of physics beyond the SM. For $\tan \beta>3$, the dominant decay mode for light charged Higgs bosons is $H^{+} \rightarrow \tau \nu_{\tau}$, for heavy charged Higgs bosons the branching fraction to $\tau \nu_{\tau}$ can still be sizable. The main production modes depend on the mass of the charged Higgs. For $m\left(H^{+}\right)<m_{t}$, it can be produced in top quark decays, while for $m\left(H^{+}\right)>m_{t}$, associated production together with top quarks takes over. The ATLAS analysis shown here ${ }^{18}$ uses $t \bar{t}$ events with a $\tau$ lepton decaying hadronically in the final state, with veto on any other leptons. The analysis requires at least three or four jets, with at least one of them b-tagged, and large missing $E_{T}$. The discriminating variable is the transverse invariant mass of the $\tau$ products combined with the missing $E_{T}$, defined as

$$
m_{T}=\sqrt{2 p_{T}^{\tau} E_{T}^{m i s s}\left(1-\cos \Delta \phi_{\tau, m i s s}\right)}
$$

where $\Delta \phi_{\tau, \text { miss }}$ is the azimuthal angle between the hadronic decay products of the $\tau$ lepton and the direction of the missing transverse momentum. Main backgrounds are general $t \bar{t}$, single top, $\mathrm{W} / \mathrm{Z}+$ jets and di-boson production, as well as $\mathrm{QCD}$. 

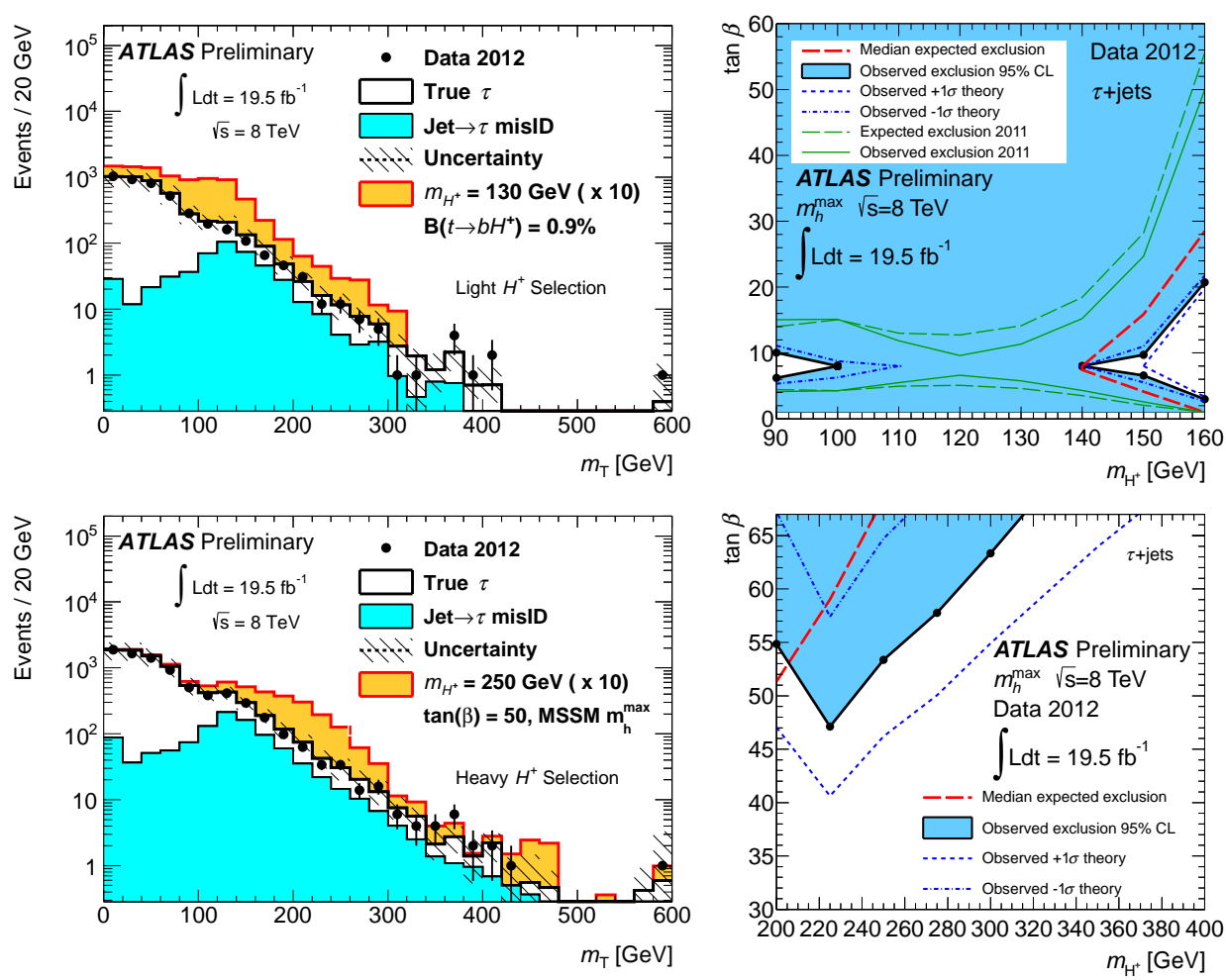

Fig. 6. The left-hand plots show the data and background predictions for the ATLAS $\mathrm{H}^{+}$boson search $\frac{18}{18}$ as function of $m_{T}$, both for the light (top) and heavy (bottom) Higgs mass selections. Expected Higgs signals for Higgs masses of 130 and $250 \mathrm{GeV}$ are also superimposed, scaled up by a factor of ten, assuming $B R\left(t \rightarrow b H^{+}\right)=0.9 \%$ and $\tan \beta=50$, respectively. The right-hand plots show the resulting model-dependent limits in the $m_{H+}$ and $\tan \beta$ parameter plane, again for the light (top) and heavy (bottom) Higgs mass selections 18 .

The full 2012 dataset is used for this measurement. The low and high mass selections cover $H^{+}$mass ranges of 90-160 and 180-600 GeV, respectively. Figure 6 shows the $m_{T}$ spectra as well as the model dependent limits for both selections. No evidence for a $\mathrm{H}^{+}$signal is found. At low masses, large parts of the MSSM parameter space are excluded, while at high masses relatively large values of $\tan \beta$ are still allowed.

\section{Generic 2HDM Searches}

While the MSSM is a very specific implementation of a Higgs sector motivated by supersymmetry, the Two-Higgs Doublet Model (2HDM) is a phenomenological approach, which allows data interpretations based on two Higgs doublets without committing to a particular theory. As opposed to MSSM at tree level, the 2HDM can accomodate $\mathrm{CP}$ violation, as well as flavor-changing couplings. Examples for 

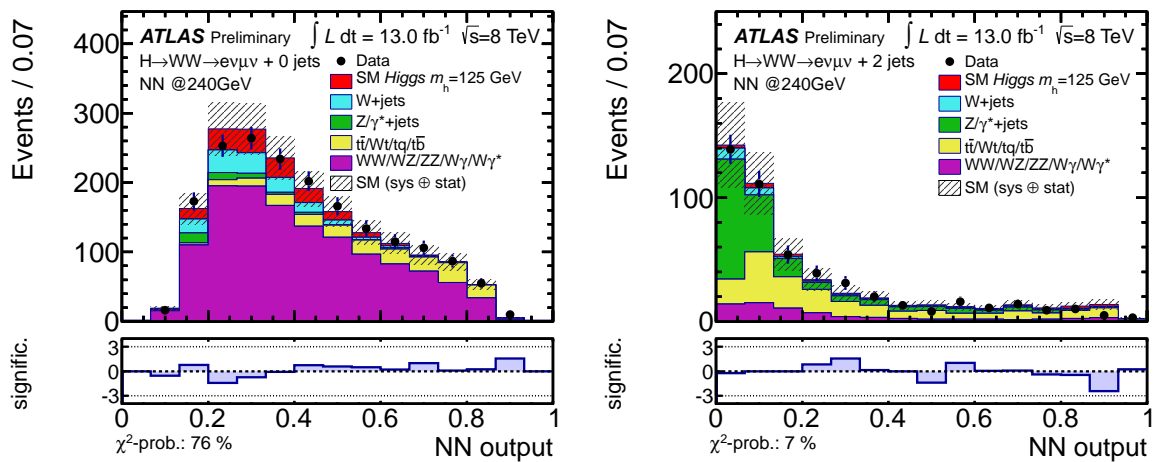

Fig. 7. Distributions of the neural network discriminant variable for the ATLAS $h / H \rightarrow$ $W W^{(*)} \rightarrow e \nu \mu \nu$ analysis 19 , optimized for a Higgs mass of $240 \mathrm{GeV}$, for the GGF (left) and VBF selections (right).

categories of $2 \mathrm{HDM}$ models with natural flavor conservation are the Type-I, in which all quarks couple only to one Higgs doublet, and the Type-II, in which the up-type quarks couple to one and the down-type quarks couple to the other Higgs doublet. Key parameters are $\tan \beta$, which is the ratio of the vacuum expectation values of the two Higgs doublets, and the scalar mixing angle $\alpha$. The MSSM is a special case of a Type-II 2HDM. As the MSSM, the 2HDM feature three neutral and two charged Higgs bosons.

A recent analysis from ATLAS ${ }^{19}$ searches for decays of the scalar Higgs bosons $\mathrm{h}$ and $\mathrm{H}$ in the channel $h / H \rightarrow W W^{(*)} \rightarrow e \nu \mu \nu$, assuming $m_{h}=125 \mathrm{GeV}$. The pseudo-scalar A does not decay into $\mathrm{W}$ pairs. The analysis requires exactly two leptons of opposite charge and missing energy. In order to cover the GGF and vector boson fusion (VBF) production mechanisms, either zero or two jets are required, respectively. A neural network combining several kinematic variables is trained for each mass point to enhance the signal over the background. The Higgs boson observed near $125 \mathrm{GeV}$ is also treated as background. The resulting distributions of the neural network discriminant are shown in Figure 7 for an assumed Higgs mass of $240 \mathrm{GeV}$. The data are compared to a model of simulated events representing the various background processes. No indication of a signal is seen. The results are translated into exclusion contours within the $2 \mathrm{HDM}$ parameter space. Figure 8 shows examples for such contours for two values of $\tan \beta$, each for Type-I and Type-II $2 \mathrm{HDM}$ models. For low masses, significant parts of the $\cos \alpha$ range are excluded.

\section{NMSSM Higgs Search}

The next-to-minimal supersymmetric model (NMSSM) features two complex Higgs doublets and an additional scalar field. The physical states are mixtures: three CPeven $\left(h_{1}, h_{2}, h_{3}\right)$, two CP-odd $\left(a_{1}, a_{2}\right)$, and two charged bosons $\left(h^{ \pm}\right)$. The NMSSM 

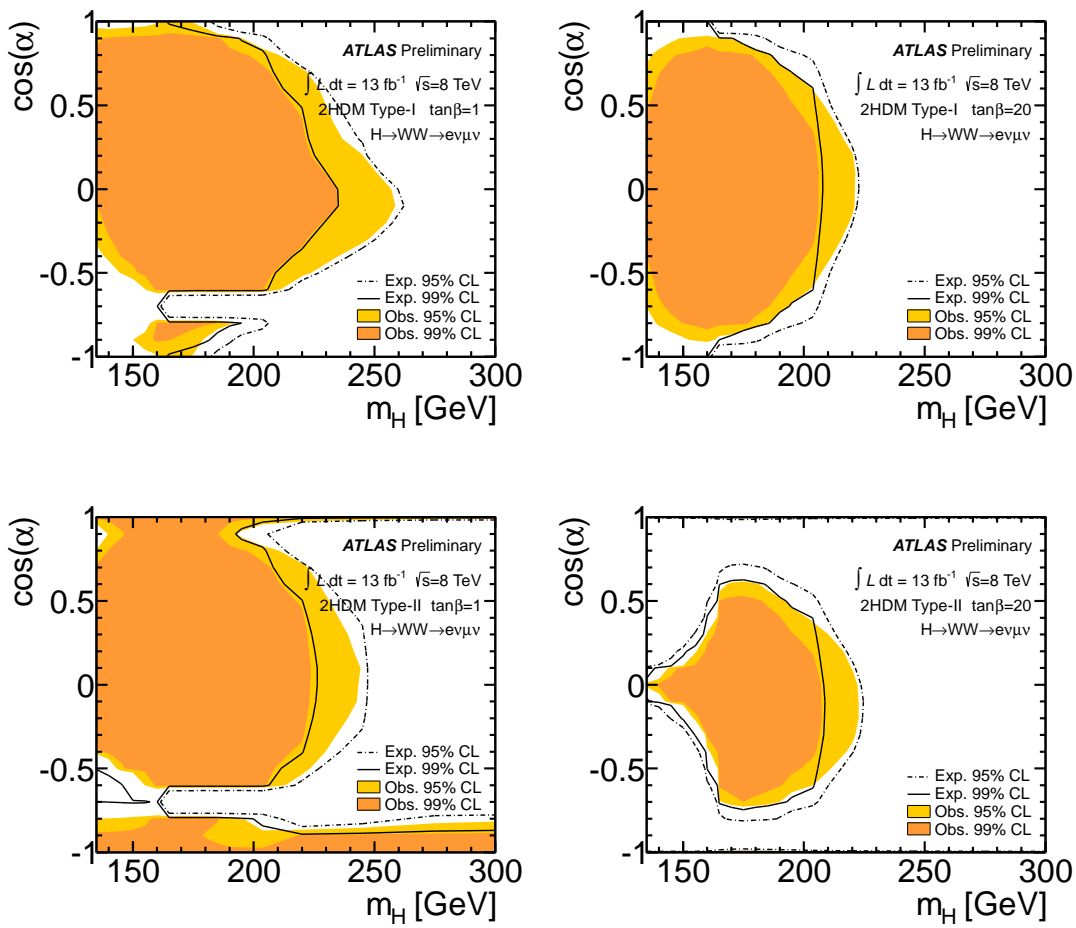

Fig. 8. Exclusion contours in the $\cos \alpha-m_{H}$ parameter space for the ATLAS $h / H \rightarrow W W^{(*)} \rightarrow$ $e \nu \mu \nu$ analysis 19 . The top row shows Type-I and the bottom row Type-II 2HDM models. The left and right column plots assume $\tan \beta=1$ and $\tan \beta=20$, respectively.

requires less fine tuning for the Higgs mass, and solves the so-called " $\mu$ problem" of the MSSM.

A recent CMS analysis 20 searches for the decay of a non-standard Higgs into two very light bosons, resulting in two boosted pairs of muons. The corresponding NMSSM interpretation is a decay chain $h_{1,2} \rightarrow a_{1} a_{1} \rightarrow(\mu \mu)(\mu \mu)$, where either the $h_{1}$ or the $h_{2}$ could correspond to the boson observed near $125 \mathrm{GeV}$, and $a_{1}$ is a new CP-odd Higgs boson lighter than twice the $\tau$ mass. But also an interpretation within dark-SUSY models is possible, based on the decay chain $h \rightarrow 2 n_{1} \rightarrow 2 n_{D}+2 \gamma_{D} \rightarrow$ $2 n_{D}+(\mu \mu)(\mu \mu)$, where $n_{1}$ is the lightest visible neutralino, $n_{D}$ is a light dark fermion and $\gamma_{D}$ a light massive dark photon with weak couplings to SM particles.

The analysis selects events with two isolated, boosted muon pairs, considering the mass ranges $0.25<m_{a}<3.55 \mathrm{GeV}$ and $m_{h}>86 \mathrm{GeV}$. The signal region is defined by requiring the masses of the two muon pairs to be equal. Main backgrounds in the analysis are direct production of $J / \Psi$ pairs, and $b \bar{b}$ production with subsequent di-muon decays, either semi-leptonic or via quarkonia resonances. The $b \bar{b}$ background is estimated from a bb-enriched control sample and the double- $J / \Psi$ 

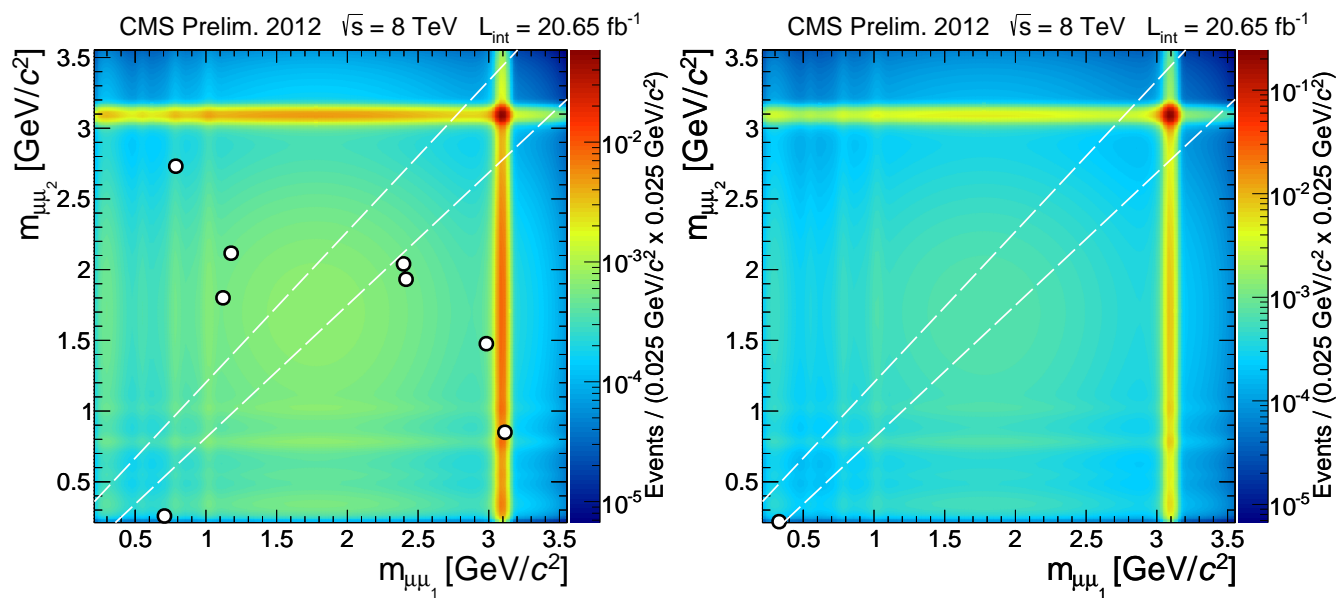

Fig. 9. Distribution in the invariant masses of the two muon pairs, in the off-diagonal control region (left) and the diagonal signal region (right) in the CMS NMSSM search 20. The empty circles show the surviving events in the data. The histogram represented by shades shows the $b \bar{b}$ background template.
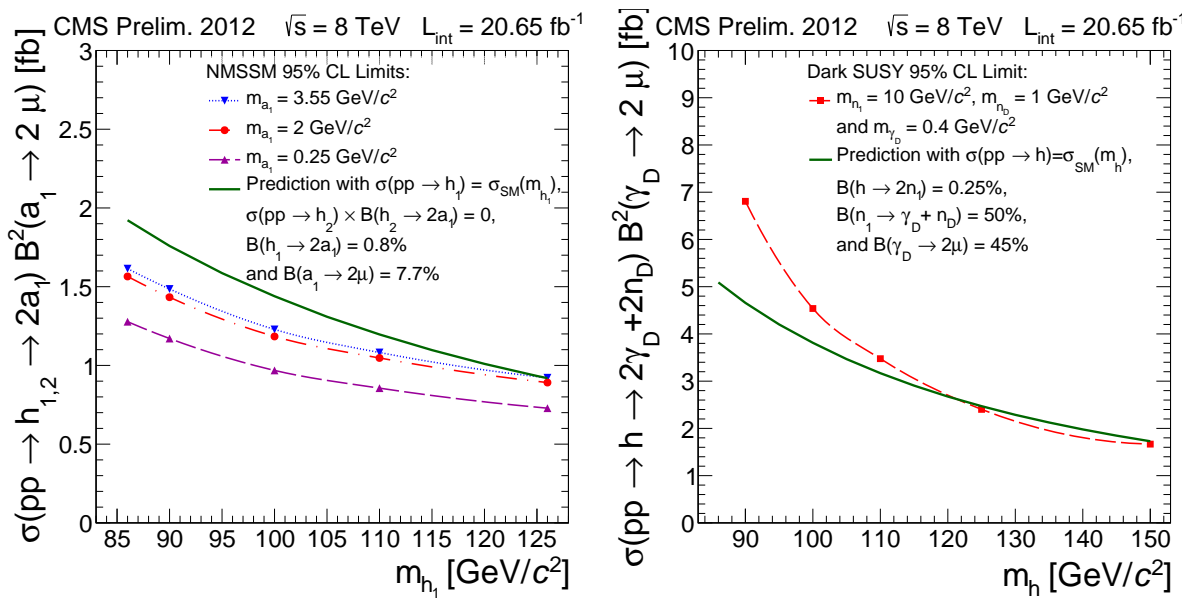

Fig. 10. Interpretation of the CMS results in the NMSSM (left) and the dark-SUSY (right) benchmark models. (See text for details).

production from PYTHIA. Figure 9 (left) shows the distribution in the space of the two di-muon masses with the diagonal signal region still blinded. Eight events are observed in the off-diagonal sideband. After unblinding, only one event is observed in the diagonal signal region (Figure 9 (right)), consistent with an expected background of $3.8 \pm 2.1$ events. The results are interpreted in the context of NMSSM and dark-SUSY models. The NMSSM interpretation (Figure 10 (left)) shows $95 \%$ 
CL upper limits on the cross section times branching fraction vs. the Higgs mass $m_{h_{1}}$ for different values of $m_{a_{1}}$. The dark-SUSY interpretation (Figure 10 (right)) displays corresponding limits vs. $m_{h}$ for a simplified scenario for $m_{n_{1}}=10 \mathrm{GeV}$ and $m_{n_{D}}=1 \mathrm{GeV}$ and $m_{\gamma_{D}}=0.4 \mathrm{GeV}$. The comparison shows that the experimental limits of this analysis are already able to exclude certain models.

\section{Searches for Invisible Higgs Bosons}

If a Higgs boson would decay with a significant probability into final states consisting entirely of invisible particles, this might still be measurable if the Higgs is produced in association with other, well detectable particles. Searches of invisible Higgs decays in association with $\mathrm{Z}$ bosons have lead to upper limits on the invisible branching fraction of $65 \%\left(\mathrm{ATLAS}^{22}\right)$ and $75 \%\left(\mathrm{CMS}^{23}\right)$ for a Higgs with SM cross section at a mass of $125 \mathrm{GeV}$ at $95 \%$ confidence level, leaving still plenty of room for such decays.
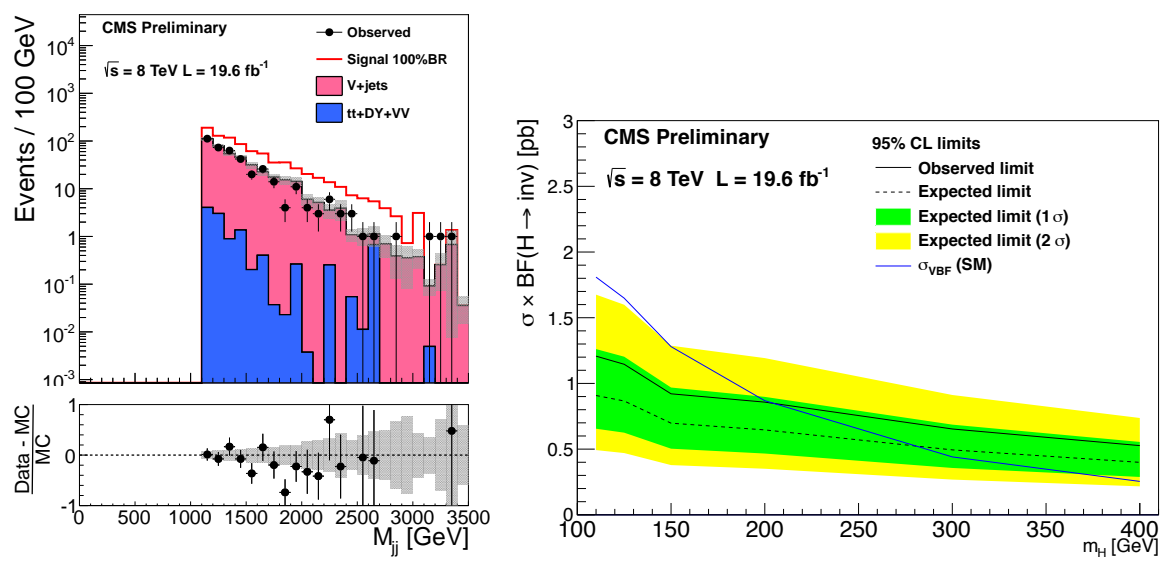

Fig. 11. Left: Di-jet mass distribution for VBF events together with the estimated backgrounds in the invisible Higgs search of $\mathrm{CMS} 21$. The signal expected for a $100 \%$ invisible branching fraction is also shown. Right: Upper limits for $\sigma \times B R$ into invisible final states. The full VBF Higgs production cross section is also shown.

Recently, invisible Higgs decays have also been searched for by CMS in VBF topology 21 , which has a higher cross section than associated $\mathrm{Z}$ production. The final state is characterized by two scattered jets with a large rapidity gap, very little other activity in the event and large missing $E_{T}$. Special triggers combining VBF and missing $E_{T}$ signatures are crucial for this analysis, and large efforts have been undertaken to reduce the QCD background. The signal is searched for in the invariant mass of the two jets. The signature of an invisible Higgs should manifest as an excess which is growing with the dijet mass. Figure 11 (left) shows the observed 
distribution in the data with the background expectation and the expected signal in case the Higgs near $125 \mathrm{GeV}$ would decay to $100 \%$ into invisible modes. No signal is observed. Figure 11 (right) shows the obtained upper limits on the cross section times branching fraction as a function of the Higgs mass, in comparison with the full VBF cross section. At $m_{H}=125 \mathrm{GeV}$, the upper limit on the invisible branching fraction is $69 \%$.

\section{Summary}

The observation of SM-like properties of the established Higgs state near $125 \mathrm{GeV}$ does not imply that the Higgs sector must have SM structure. The best way of clarification is the direct search for additional Higgs signatures.

A broad attack is launched to clarify whether the Higgs sector reaches beyond the SM. In the context of MSSM, at low masses $m_{A}$ the limits from LEP and LHC start to close. Large values of $m_{A}$ and $\tan \beta$ are still possible. The constraints from the $H^{+}$searches have significantly improved. Recently, analyses interpret their findings also in the 2HDM approach. Concerning the NMSSM, only few channels have been targeted so far, and there is still a wide open range of possibilities. The search for invisible Higgs particles and decay modes yields first results in vector boson associated production and VBF signatures, but the limits on the invisible branching fraction are still large.

In summary, the Higgs searches beyond the SM have just scratched the surface. Many LHC analyses are being updated with the full $8 \mathrm{TeV}$ statistics, and the Run-II of the LHC at $\approx 13 \mathrm{TeV}$ will further extend the reach towards higher masses.

\section{References}

1. ATLAS Collab., Phys. Lett. B716 (2012) 1.

2. CMS Collab., Phys. Lett. B716 (2012) 30.

3. ATLAS Collab., J. Inst. 3 (2008) S08003.

4. CMS Collab., J. Inst. 3 (2008) S08004.

5. E. Witten, Phys. Lett B105 (1981) 267.

6. CMS Collab., CMS PAS HIG-13-005 (2013).

7. M. Carena et al., Eur. Phys. J. C26 (2003) 601;

M. Carena et al., Eur. Phys. J. C45 (2006) 797.

8. M. Carena et al., arXiv:1302.7033 (2013).

9. P. Bechtle et al., arXiv:1211.1955 (2012).

10. D0 Collab., Phys. Lett. B710 (2012) 569;

CDF Collab., Phys. Rev. Lett. 103 (2009) 201801.

11. ATLAS Collab., JHEP 02 (2013) 095.

12. CMS Collab., CMS PAS HIG-12-050 (2012).

13. A. Elagin et al., Nucl. Instr. Meth. A654 (2011) 481.

14. CDF Collab., Phys. Rev. D85 (2012) 032005.

15. D0 collab., arXiv:1011.1931, Phys. Lett. B698 (2011) 97.

16. CDF and D0 Collab., arXiv:1207.2757, Phys. Rev. D86 (2012) 091101.

17. CMS Collab., Phys. Lett. B722 (2013) 207.

18. ATLAS Collab., ATLAS CONF-2013-090 (2013). 
19. ATLAS Collab., ATLAS CONF-2013-027 (2013).

20. CMS Collab., CMS PAS HIG-13-010 (2013).

21. CMS Collab., CMS PAS HIG-13-013 (2013).

22. ATLAS Collab., ATLAS CONF-2013-011 (2013).

23. CMS Collab., CMS PAS HIG-13-018 (2013). 